\title{
Iodocyclisation of $N$-allyl ureas; a route to imidazolin-2-ones
}

\author{
Christopher J. Moody, ${ }^{\text {a,b* }}$ Peter A. Hunt, ${ }^{b}$ and Colin Smith ${ }^{c}$ \\ ${ }^{a}$ School of Chemistry, University of Exeter, Stocker Road, Exeter EX4 4QD \\ ${ }^{b}$ Department of Chemistry, Imperial College, London SW7 2AY \\ ${ }^{c}$ GlaxoWellcome Research \& Development, Medicines Research Centre, Gunnels Wood \\ Road, Stevenage, Hertfordshire SG1 2NY \\ *University of Exeter; fax 441392 263434; \\ E-mail: c.j.moody@ex.ac.uk
}

\section{Dedicated to Professor Otto Meth-Cohn on the occasion of his $65^{\text {th }}$ birthday \\ (received 14 May 00; accepted 03 Oct 00; published on the web 11 Oct 00)}

\begin{abstract}
Treatment of $N$-allyl ureas 7-9 with TMS triflate, followed by iodine in THF resulted in iodocyclisation to give the imidazolin-2-ones 10-12 in good yield.
\end{abstract}

Keywords: Iodocyclisation, urea, imidazolinone, imidazole, hydantoin

\section{Introduction}

The activation of a $\mathrm{C}=\mathrm{C}$ double bond by an external electrophile $\mathrm{E}^{+}$, followed by addition of an internal heteroatom nucleophile is not only a useful method for the construction of heterocyclic compounds but also for cyclofunctionalisation of the original $\mathrm{C}=\mathrm{C}$ bond. ${ }^{1,2}$ Although, in general, there is a preference for exo-cyclisation, the endo-mode of cyclisation is not precluded. However, in cases where other nucleophilic heteroatoms are present in the precursor, either or both heteroatoms can participate in the cyclisation (Scheme 1).
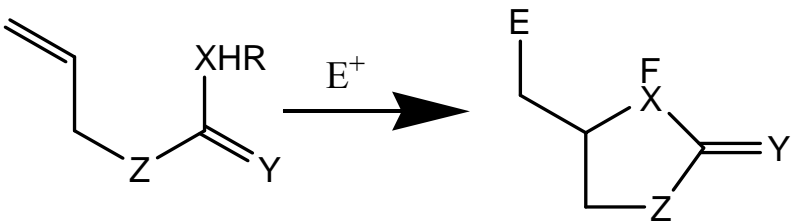

and/or

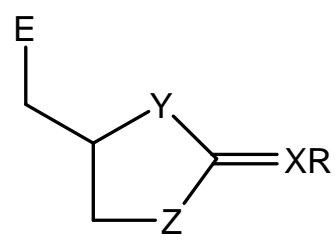

\section{Scheme 1}


In connection with a projected synthesis of analogues of nikkomycin $\mathrm{X}$, we required a route to 4-formyl-1-alkylimidazolin-2-ones, and were attracted by the possibility of using an electrophile mediated cyclisation of an appropriate $N$-allyl urea 1 (Scheme 2). Since the publication of our preliminary communication, ${ }^{2}$ several other reports on the cyclisation reactions of $N$-allyl ureas and thioureas have appeared. ${ }^{3-8}$<smiles></smiles>
nikkomycin X

\section{Scheme 2}

\section{Results and Discussion}

The substrates for the iodocyclisation reactions were the $N$-allyl ureas 6-9, prepared from the corresponding allylamines 2-5. Allylamine 2 and $N$-methylallylamine 3 are commercially available, and the $N$-benzyl- and $N$-cyclopentyl derivatives 4 and 5 were readily obtained from allylamine 2 by reductive amination of benzaldehyde and cyclopentanone respectively using sodium cyanoborohydride as reducing agent at $\sim \mathrm{pH} 6 .{ }^{9}$ Reaction of the allylamines 2-5 with trimethylsilyl isocyanate gave the required ureas 6-9 in good yield (Scheme 3). ${ }^{10}$

In order to ensure that the iodocyclisation of the $N$-allyl ureas proceeded via nitrogen, rather than oxygen, we followed the procedure developed by Knapp for cyclisation of related unsaturated amides. ${ }^{11}$ Thus treatment of the ureas with trimethylsilyl trifluoromethanesulfonate and triethylamine, was followed by reaction with iodine in THF. Although no cyclised products could be isolated from reaction of the $N$-allyl urea 6 under the above conditions, the ureas 7-9 cyclised to give the corresponding 4-iodomethylimidazolin-2ones 10-12 in good yield (Scheme 3). 




\section{Scheme 3}

The chemistry of the 4-iodomethylimidazolin-2-one 12 was briefly investigated (Scheme 4). In parallel with Knapp's observations on iodomethyl lactams, ${ }^{12}$ we found that the iodide 12 was relatively unreactive towards nucleophilic displacement. Also attempted oxidation to the aldehyde using dimethylsulfoxide or trimethylamine- $N$-oxide proved unsuccessful. However silver(I) assisted hydrolysis proceeded smoothly and gave the alcohol 13, as an unstable oil which could not be purified. Although various attempt to oxidise 13 to the desired aldehyde were unsuccessful, the use of cerium ammonium nitrate as oxidant resulted in relatively clean oxidation to the hydantoin 14 (Scheme 4). Finally, on treatment with base, the iodomethyl compound 12 readily underwent elimination to give, after double bond isomerisation, the 4methylimidazol-2-one 15 in excellent yield.
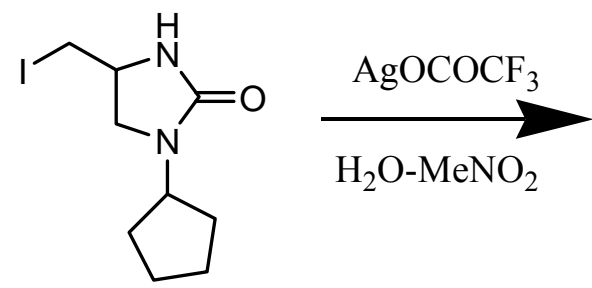

12

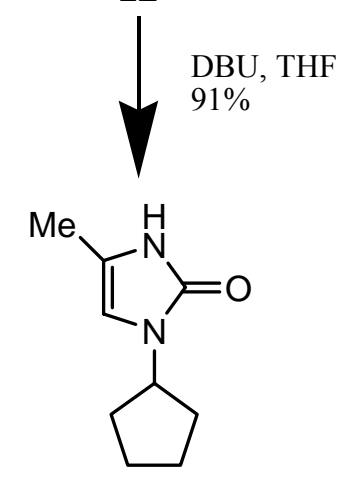

15<smiles>O=C1NC(CO)CN1C1CCCC1</smiles>

13

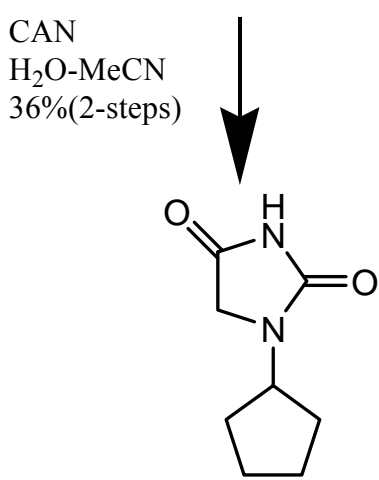

14

\section{Scheme 4}




\section{Experimental Section}

General Procedures. Tetrahydrofuran was distilled from potassium benzophenone ketyl and stored under nitrogen or used immediately. Dichloromethane was distilled from phosphorus pentoxide and used under nitrogen. Light petroleum (bp 40-60 ${ }^{\circ} \mathrm{C}$ ) was distilled after purchase and then stored normally. All remaining reagents and chromatography solvents were used as purchased, without further purification. Flash column chromatography was carried out on Merck silica gel $60 \mathrm{H}$.

Infrared spectra were recorded on a Perkin-Elmer model 1710 infrared Fourier transform spectrometer. Proton nuclear magnetic resonance spectra were recorded on either a Bruker WM $250(250 \mathrm{MHz})$, or a Jeol GSX $270(270 \mathrm{MHz})$ spectrometer. Chemical shifts are reported in parts per million downfield of tetramethylsilane by referencing to tetramethylsilane itself or the residual proton resonances of the solvents as the appropriate internal standard. Carbon-13 nuclear magnetic resonance spectra were recorded on either a Bruker WM $250(62.5 \mathrm{MHz})$, or a Jeol GSX $270(67.5 \mathrm{MHz})$ spectrometer and were referenced to the solvent.

Electron ionisation mass spectra were performed on either A.E.I. MS 12 or VG Micromass $7070 \mathrm{~B}$ instruments and run at either 14 or $70 \mathrm{eV}$ source potentials. All accurate mass determinations were performed by the Mass Spectrometry Service Centre at the Swansea EPSRC.

$N$-Alkylallylamines 4 and 5. To a solution of anhydrous allylamine $2(60 \mathrm{mmol})$ in absolute methanol $(25 \mathrm{ml})$ was added methanolic $\mathrm{HCl}(5 \mathrm{M} ; 2.5 \mathrm{ml})$ followed by the aldehyde or ketone $(10 \mathrm{mmol})$ and $\mathrm{NaBH}_{3} \mathrm{CN}(300 \mathrm{mg}, 5 \mathrm{mmol})$. The mixture was then stirred for about $90 \mathrm{~h}$ at room temperature. Concentrated $\mathrm{HCl}$ was added until the $\mathrm{pH}<2$ and the methanol was removed under reduced pressure. The residue was taken up in water $(10 \mathrm{ml})$ and extracted with ether $(3 \times 20 \mathrm{ml})$. The aqueous layer was taken to $\mathrm{pH}>10$ using solid $\mathrm{KOH}$, saturated with $\mathrm{NaCl}$ and then extracted again with ether $(3 \times 20 \mathrm{ml})$. The ether extracts were dried $\left(\mathrm{MgSO}_{4}\right)$, filtered and concentrated to give a brown oil which was purified by column chromatography (eluant light petroleum to ether) to give the $\mathrm{N}$-alkylallylamines.

$N$-Benzylallylamine 4. Prepared from allylamine $2(4.5 \mathrm{ml}, 60 \mathrm{mmol})$, benzaldehyde $(1.0 \mathrm{ml}$, $10 \mathrm{mmol}$ ) and $\mathrm{NaBH}_{3} \mathrm{CN}$ (300 mg, $5 \mathrm{mmol}$ ) according to the above method. Isolated as a pale oil $(75 \%)$, bp $40{ }^{\circ} \mathrm{C}$ at $0.5 \mathrm{mmHg}$ (lit., ${ }^{13}$ bp $40-41{ }^{\circ} \mathrm{C}$ at $0.1 \mathrm{mmHg}$ ); $v_{\max }$ (neat) 3310,3064 , $3028,2979,2824,1644,1495,1454,1106,1028,995,919,737,699 \mathrm{~cm}^{-1} ; \delta_{\mathrm{H}}(250 \mathrm{MHz}$; $\left.\mathrm{CDCl}_{3}\right) 1.95(1 \mathrm{H}$, br s, $\mathrm{NH}), 3.3\left(2 \mathrm{H}, \mathrm{dt}, \mathrm{NCH}_{2} \mathrm{CH}=\mathrm{CH}_{2}, \mathrm{~J}\right.$ 6.0, $\left.1.3 \mathrm{~Hz}\right), 3.8(2 \mathrm{H}, \mathrm{s}$, $\mathrm{NCH}_{2} \mathrm{Ph}$ ), 5.1 (1 H, ddd, $\left.\mathrm{CH}=\mathrm{CHH}, \mathrm{J} 10.5,3.0,1.3 \mathrm{~Hz}\right), 5.2(1 \mathrm{H}, \mathrm{ddd}, \mathrm{CH}=\mathrm{CHH}, \mathrm{J} 17.0,3.0$, $1.3 \mathrm{~Hz}), 5.9\left(1 \mathrm{H}\right.$, ddt, $\mathrm{NCH}_{2} \mathrm{CH} \mathrm{CH}_{2}, \mathrm{~J}$ 17.0, 10.5, $\left.6.0 \mathrm{~Hz}\right), 7.2-7.4(5 \mathrm{H}, \mathrm{m}, \mathrm{Ph}) ; \mathrm{m} / \mathrm{z} 147$ $\left(M^{+}, 6 \%\right), 146(8), 105(4), 91(31), 56(10), 41(6), 28(100)$. 
$\mathrm{N}$-Cyclopentylallylamine 5. Prepared from allylamine $2(18 \mathrm{ml}, 240 \mathrm{mmol})$, cyclopentanone (3.5 ml, $40 \mathrm{mmol})$ and $\mathrm{NaBH}_{3} \mathrm{CN}(1.20 \mathrm{~g}, 19 \mathrm{mmol})$ according to the above method. Isolated as a clear colourless viscous oil (85\%), bp $60{ }^{\circ} \mathrm{C}$ at $50 \mathrm{mmHg}$ (Kugelrohr) (lit., ${ }^{14}$ bp $60-70{ }^{\circ} \mathrm{C}$ at $15 \mathrm{mmHg}$ ); $v_{\max }$ (neat) $3284,1644 \mathrm{~cm}^{-1} ; \delta_{\mathrm{H}}\left(250 \mathrm{MHz} ; \mathrm{CDCl}_{3}\right) 1.2-1.9(8 \mathrm{H}, \mathrm{m}$, cyclopentyl $\left.\mathrm{CH}_{2}\right), 3.05(1 \mathrm{H}, \mathrm{q}, \mathrm{NCH}, \mathrm{J} 6.8 \mathrm{~Hz}), 3.15(2 \mathrm{H}, \mathrm{dd}, \mathrm{CH} 2 \mathrm{CH}=\mathrm{CH} 2, \mathrm{~J} 5.9,1.5 \mathrm{~Hz}), 5.0(1 \mathrm{H}$, ddd, $\left.\mathrm{CH}_{2} \mathrm{CH}=\mathrm{CHH}, \mathrm{J} 10.3,3.5,1.5 \mathrm{~Hz}\right), 5.1\left(1 \mathrm{H}\right.$, ddd, $\left.\mathrm{CH}_{2} \mathrm{CH}=\mathrm{CHH}, \mathrm{J} 17.1,3.5,1.5 \mathrm{~Hz}\right)$, $5.85\left(1 \mathrm{H}, \mathrm{m}, \mathrm{CH}_{2} \mathrm{CH}=\mathrm{CH}_{2}, \mathrm{~J} 17.1,10.3,5.9 \mathrm{~Hz}\right) ; \mathrm{m} / \mathrm{z} 125\left(M^{+}, 19 \%\right), 96$ (88), 82 (18), 68 (22), 56 (14), 41 (44).

$\mathrm{N}$-Allylurea 6. Allylamine $2(0.7 \mathrm{ml}, 10 \mathrm{mmol})$ was added, via syringe, to dry benzene (4 ml) under nitrogen and trimethylsilyl isocyanate (TMSNCO, $1.35 \mathrm{ml}, 10 \mathrm{mmol}$ ) was added to the stirred solution. The solution was heated under reflux for $1 \mathrm{~h}$ and after cooling, the product partially precipitated from the solution as a colourless solid and was filtered off. The remaining product was obtained after the solvent was evaporated to give the title compound $(0.85 \mathrm{~g}, 85 \%)$ as platelets, $\mathrm{mp} 85-87^{\circ} \mathrm{C}$, (lit., ${ }^{10} \mathrm{mp} 85^{\circ} \mathrm{C}$ ); $v_{\max }$ (Nujol) 3436, 3334, $1652 \mathrm{~cm}^{-1}$; $\delta_{\mathrm{H}}\left(250 \mathrm{MHz} ; \mathrm{CDCl}_{3}\right), 3.75\left(2 \mathrm{H}\right.$, br t, $\left.\mathrm{CH}_{2} \mathrm{~N}, \mathrm{~J} 5.3 \mathrm{~Hz}\right), 4.6\left(3 \mathrm{H}, \mathrm{br} \mathrm{s}, \mathrm{NH}\right.$ and $\left.\mathrm{NH}_{2}\right), 5.1(1$ $\mathrm{H}$, ddt, $\mathrm{HCH}=\mathrm{CH}$, J 10.5, 3.8, $1.5 \mathrm{~Hz}), 5.19$ (1 H, ddt, $\mathrm{HCH}=\mathrm{CH}, \mathrm{J} 17.6,3.8,1.5 \mathrm{~Hz}), 5.83(1$ $\mathrm{H}$, ddt, $\mathrm{CH}_{2}=\mathrm{CH}, \mathrm{J}$ 10.5, 7.6, $5.3 \mathrm{~Hz}$ ); m/z 100 (M+, 5\%), 85 (1), 57 (100), 56 (58), 44 (20).

$N$, $N$-Methylallylurea 7. $N$-Methylallylamine $3(0.5 \mathrm{ml}, 5.5 \mathrm{mmol})$ was added via a syringe to dry benzene $(10 \mathrm{ml})$ under nitrogen and TMSNCO $(0.8 \mathrm{ml}, 6 \mathrm{mmol})$ was added to the stirred solution. The solution appeared cloudy after the addition but was heated under reflux for $1 \mathrm{~h}$ before the mixture was allowed to cool and the partially precipitated product was removed by filtration. The solvent was removed under reduced pressure and the remainder of the product was obtained. Recrystallisation from a $40-60{ }^{\circ} \mathrm{C}$ light petroleum:benzene system gave the title compound $\left(0.627 \mathrm{~g}, 87 \%\right.$ ) as a colourless solid, mp 98-99 ${ }^{\circ} \mathrm{C}$; (Found: C, 52.6; H, 8.9; N, 24.8; $\mathrm{C}_{5} \mathrm{H}_{10} \mathrm{~N}_{2} \mathrm{O}$ requires $\mathrm{C}, 52.6 ; \mathrm{H}, 8.8 ; \mathrm{N}, 24.6 \%$ ); $v_{\max }$ (Nujol) 3416, 3 357, 3201, 1657, 1609, 1298, 1101, 1046, 999, 949, 923, 774, 661, $606 \mathrm{~cm}^{-1} ; \delta_{\mathrm{H}}\left(250 \mathrm{MHz} ; \mathrm{CDCl}_{3}\right), 2.83(3 \mathrm{H}$, s, NMe), $3.8\left(2 \mathrm{H}, \mathrm{dt}, \mathrm{NCH}_{2} \mathrm{CH}=\mathrm{CH}_{2}, \mathrm{~J} 5.3,1.5 \mathrm{~Hz}\right), 4.85\left(2 \mathrm{H}\right.$, br s, $\left.\mathrm{NH}_{2}\right), 5.10(1 \mathrm{H}$, dd, $\mathrm{CH} H=\mathrm{CH}, \mathrm{J} 5.2,1.5 \mathrm{~Hz}), 5.15(1 \mathrm{H}, \mathrm{dd}, \mathrm{CHH}=\mathrm{CH}, \mathrm{J} 3.0,1.5 \mathrm{~Hz}), 5.73\left(1 \mathrm{H}, \mathrm{ddt}, \mathrm{CH}_{2}=\mathrm{CH}, \mathrm{J}\right.$ 5.2, 3.0, $5.3 \mathrm{~Hz}) ; \mathrm{m} / \mathrm{z} 114\left(M^{+}, 41 \%\right), 99$ (4), 71 (100), 70 (55), 56 (27), 44 (91), 41 (41).

$N, N$-Benzylallylurea 8. $N$-Benzylallylamine $4(125.4 \mathrm{mg}, 0.85 \mathrm{mmol})$ was dissolved in dry benzene $(5 \mathrm{ml})$ under nitrogen and TMSNCO $(0.23 \mathrm{ml}, 1.7 \mathrm{mmol})$ was added to the stirred solution. The mixture was heated under reflux for $24 \mathrm{~h}$ before the solvent was removed under vacuum to give a pale oil. This oil was purified by chromatography (eluant light petroleum to ether to EtOAc) and gave the title compound (124 mg, 77\%) as a colourless viscous oil, bp $174{ }^{\circ} \mathrm{C}$ at $0.4 \mathrm{mmHg}$; (Found: $\mathrm{C}, 69.5 ; \mathrm{H}, 7.6 ; \mathrm{N}, 14.9 ; \mathrm{C}_{11} \mathrm{H}_{14} \mathrm{~N}_{2} \mathrm{O}$ requires $\mathrm{C}, 69.4 ; \mathrm{H}, 7.4$, $\mathrm{N}, 14.7 \%$ ); $v_{\max }$ (Nujol) 3482, 3354, 3210, 1651, 1599, 1360, 1317, 1295, 1077, 964, 924, 734, $700 \mathrm{~cm}^{-1} ; \delta_{\mathrm{H}}\left(250 \mathrm{MHz} ; \mathrm{CDCl}_{3}\right), 3.84\left(2 \mathrm{H}, \mathrm{d}, \mathrm{CH}_{2}=\mathrm{CHCH}_{2} \mathrm{~N}, \mathrm{~J} 4.8 \mathrm{~Hz}\right), 4.48(2 \mathrm{H}, \mathrm{s}$, $\left.\mathrm{NCH}_{2} \mathrm{Ph}\right), 4.7\left(2 \mathrm{H}\right.$, br s, $\left.\mathrm{NH}_{2}\right), 5.15(1 \mathrm{H}$, br s, $\mathrm{CH}=\mathrm{CHH}), 5.21(1 \mathrm{H}$, br d, $\mathrm{CH}=\mathrm{CHH}$, J 3.5 
$\mathrm{Hz}), 5.75\left(1 \mathrm{H}, \mathrm{m}, \mathrm{CH}_{2}=\mathrm{CH}\right), 7.2-7.4(5 \mathrm{H}, \mathrm{m}, \mathrm{Ph}) ; \mathrm{m} / \mathrm{z} 190\left(M^{+}, 27 \%\right), 149$ (36), 122 (32), 106 (78), 91 (100), 77 (31), 56 (37), 41 (30).

$\mathrm{N}, \mathrm{N}$-Cyclopentylallylurea 9. $\mathrm{N}$-Allylcyclopentylamine $5(1.54 \mathrm{~g}, 12.3 \mathrm{mmol})$ was taken up in dry THF $(30 \mathrm{ml})$ and the solution placed under nitrogen. TMSNCO $(3.3 \mathrm{ml}, 24.6 \mathrm{mmol})$ was added to the stirred solution before the mixture was heated under reflux for $24 \mathrm{~h}$. The solvent was removed under reduced pressure and the residue was chromatographed using chromatography (eluant light petroleum to ether to EtOAc). The title compound $(1.96 \mathrm{~g}, 95 \%)$ was isolated as a colourless viscous oil, bp $110{ }^{\circ} \mathrm{C}$ at $30 \mathrm{mmHg}$; (Found: $\mathrm{M}^{+}, 168.1263$. $\mathrm{C}_{9} \mathrm{H}_{16} \mathrm{~N}_{2} \mathrm{O}$ requires 168.1263); $v_{\max }\left(\mathrm{CHCl}_{3}\right) 3508,3414,3211,1706,1652,1588 \mathrm{~cm}^{-1}$; $8 \mathrm{H}(270$ мHz; $\left.\mathrm{CDCl}_{3}\right), 1.2-1.9\left(8 \mathrm{H}, \mathrm{m}\right.$, cyclopentyl $\left.\mathrm{CH}_{2}\right), 3.7\left(2 \mathrm{H}, \mathrm{m}, \mathrm{CH}_{2}=\mathrm{CHCH}_{2} \mathrm{~N}\right), 4.6(1 \mathrm{H}, \mathrm{q}$, $\mathrm{NCH}, \mathrm{J} 8.1 \mathrm{~Hz}), 4.75\left(2 \mathrm{H}\right.$, br s, $\left.\mathrm{NH}_{2}\right), 5.2-5.3\left(2 \mathrm{H}, \mathrm{m}, \mathrm{CH}=\mathrm{CH}_{2}\right), 5.8\left(1 \mathrm{H}, \mathrm{m}, \mathrm{CH}=\mathrm{CH}_{2}\right)$; $\mathrm{m} / \mathrm{z} 168\left(M^{+}, 43 \%\right), 127,96(81), 84(60), 68$ (35), 56 (57), 41 (100).

\section{4,5-Dihydro-1-alkyl-4-(iodomethyl)imidazolin-2-one 10-12. General Method}

The allylurea 7-9 $(1 \mathrm{mmol})$ was dissolved in freshly distilled dichloromethane $(5 \mathrm{ml})$, under nitrogen, which contained a crystal of DMAP. Triethylamine $(0.30 \mathrm{ml}, 2.2$ equiv. $)$ was added via a syringe followed by trimethylsilyl triflate $(0.39 \mathrm{ml}, 2.0$ equiv. $)$. This mixture was stirred for $25 \mathrm{~min}$ at room temperature before the solvent was removed under reduced pressure. A solution of iodine $(0.508 \mathrm{~g}, 2.0 \mathrm{mmol})$ in dry THF $(7 \mathrm{ml})$ was added to the oily residue under nitrogen and this mixture was also stirred at room temperature for a further $20 \mathrm{~min}$. The THF solution was poured into $\mathrm{Na}_{2} \mathrm{~S}_{2} \mathrm{O}_{3}$ solution $(20 \% ; 20 \mathrm{ml})$ and extracted with EtOAc $(3 \times 20$ $\mathrm{ml})$. After drying $\left(\mathrm{MgSO}_{4}\right)$ and evaporating the extracts the crude solid product was purified by wet flash chromatography (eluant light petroleum to ether to EtOAc) to give the title compounds.

4,5-Dihydro-1-methyl-4-(iodomethyl)imidazolin-2-one 10. Prepared in $83 \%$ yield from $\mathrm{N}, \mathrm{N}$-methylallylurea $7(0.114 \mathrm{~g}, 1 \mathrm{mmol})$ as described above; a colourless solid, mp 113-114 ${ }^{\circ} \mathrm{C}$; (Found: C, 25.2; H, 3.5; N, 11.7. $\mathrm{C}_{5} \mathrm{H}_{9} \mathrm{IN}_{2} \mathrm{O}$ requires C, 25.0; H, 3.8; N, 11.7\%); $v_{\max }$ $\left(\mathrm{CHCl}_{3}\right) 3249,1698,756 \mathrm{~cm}^{-1} ; \delta_{\mathrm{H}}\left(250 \mathrm{MHz} ; \mathrm{CDCl}_{3}\right), 2.78(3 \mathrm{H}, \mathrm{s}, \mathrm{NMe}), 3.12(1 \mathrm{H}, \mathrm{dd}, 5-\mathrm{H}$, J 5.2, 9.2 Hz), 3.17 (2 H, d, CH 2 I, J 6.3 Hz), $3.57(1 \mathrm{H}, \mathrm{dd}, 5-\mathrm{H}, \mathrm{J} 9.2 \mathrm{~Hz}), 3.87(1 \mathrm{H}, \mathrm{m}, 4-\mathrm{H})$, $4.87(1 \mathrm{H}$, br s, $\mathrm{NH}) ; \delta_{\mathrm{C}}\left(67.5 \mathrm{MHz} ; \mathrm{CDCl}_{3}\right) 9.5(6-\mathrm{C}), 29.8\left(\mathrm{CH}_{3}-\mathrm{N}\right), 50.4(5-\mathrm{C}), 53.0(4-\mathrm{C})$; 2-C unobserved; $m / z 240$ ( $\left.\mathrm{M}^{+}, 21 \%\right), 99$ (100), 44 (12).

4,5-Dihydro-1-benzyl-4-(iodomethyl)imidazolin-2-one 11. Prepared in 73\% yield from $\mathrm{N}, \mathrm{N}$-benzylallylurea $8(0.143 \mathrm{~g}, 0.755 \mathrm{mmol})$ as described above; a colourless solid, mp 134$135^{\circ} \mathrm{C}$; (Found: $\mathrm{C}, 41.7 ; \mathrm{H}, 3.9 ; \mathrm{N}, 8.8 . \mathrm{C}_{11} \mathrm{H}_{13} \mathrm{IN}_{2} \mathrm{O}$ requires $\mathrm{C}, 41.8 ; \mathrm{H}, 4.1 ; \mathrm{N}, 8.9 \%$ ); $v_{\max }$ $\left(\mathrm{CHCl}_{3}\right) 3444,1700 \mathrm{~cm}^{-1} ; \delta_{\mathrm{H}}\left(250 \mathrm{MHz} ; \mathrm{CDCl}_{3}\right) 2.98(1 \mathrm{H}, \mathrm{dd}, 5-\mathrm{H}, \mathrm{J}$ 9.0, $5.5 \mathrm{~Hz}), 3.05-3.1$ $(1 \mathrm{H}, \mathrm{dd}, \mathrm{CHHI}, \mathrm{J} 9.5,7.0 \mathrm{~Hz}), 3.1-3.2(1 \mathrm{H}, \mathrm{dd}, \mathrm{CHHI}, \mathrm{J} 9.5,6.0 \mathrm{~Hz}), 3.43(1 \mathrm{H}, \mathrm{dd}, 5-\mathrm{H}$, J9.0 Hz), $3.85(1 \mathrm{H}, \mathrm{m}, 4-\mathrm{H}), 4.34\left(2 \mathrm{H}, \mathrm{dd}, \mathrm{NCH}_{2} \mathrm{Ph}, \mathrm{J} 15.0 \mathrm{~Hz}\right), 5.35(1 \mathrm{H}$, br s, NH), $7.3(5$ 
$\mathrm{H}, \mathrm{m}, \mathrm{Ph}) ; \delta \mathrm{C}(62.9 \mathrm{MHz} ; \mathrm{CDCl} 3) 10.1\left(\mathrm{CH}_{2} \mathrm{I}\right), 47.1\left(\mathrm{CH}_{2} \mathrm{Ph}\right), 50.4\left(\mathrm{CH}_{2} \mathrm{NBz}\right), 50.7(\mathrm{CHN}), 127.4$ (Ph-p-C), 127.8 (Ph-o-C), 128.5 (Ph-m-C), 161.2 (C=O); m/z 316 (M+15\%), 190 (4), 175 (3), $91(27), 28$ (100).

4,5-Dihydro-1-cyclopentyl-4-(iodomethyl)imidazolin-2-one 12. Prepared in 67\% yield from $N, N$-cyclopentylallylurea $9(1.22 \mathrm{~g}, 7.7 \mathrm{mmol})$ as described above; a colourless solid, mp 96$97{ }^{\circ} \mathrm{C}$. (Found: C, 36.9; H, 5.2; N, 9.4. $\mathrm{C}_{9} \mathrm{H}_{15} \mathrm{IN}_{2} \mathrm{O}$ requires $\mathrm{C}, 36.8 ; \mathrm{H}, 5.1 ; \mathrm{N}, 9.5 \%$ ); vmax $(\mathrm{CHCl}) 3216$ and $1698 \mathrm{~cm}^{-1} ; \delta_{\mathrm{H}}\left(250 \mathrm{MHz} ; \mathrm{CDCl}_{3}\right)$ 1.3-1.9 $\left(8 \mathrm{H}, \mathrm{m}\right.$, cyclopentyl $\left.\mathrm{CH}_{2}\right), 3.06(1$ H, d, 5-H, J 10.1 Hz), 3.09 (1 H, dd, CHHI, J 8.9 Hz), 3.18 (1 H, dd, 5-H, J 4.7, $10.1 \mathrm{~Hz}), 3.5$ (1 H, dd, CHHI, J 8.9 Hz), 3.8 (1 H, m, 4-H), 4.2 (1 H, q, CHN, J $8.3 \mathrm{~Hz}), 6.0$ (1 H, br s, NH); m/z 294 ( $\left.M^{+}, 82 \%\right), 265$ (100), 226 (42), 153 (36), 99 (24), 85 (64), 41 (69).

1-Cyclopentylhydantoin 14. The iodomethylimidazolone $12(512 \mathrm{mg}, 1.74 \mathrm{mmol})$ was dissolved in nitromethane $(15 \mathrm{ml})$ and water $(31.3 \mu 1,1$ equiv.) was added. This mixture was stirred and cooled to $0{ }^{\circ} \mathrm{C}$ in an ice bath, whereupon silver trifluoroacetate $(577 \mathrm{mg}, 1.5$ equiv.) was added to it in one portion. The mixture was left to stir at $0^{\circ} \mathrm{C}$ for $3.5 \mathrm{~h}$ after which time a precipitate had formed (presumably AgI). The precipitate was filtered off using a layer of Celite and washed with EtOAc $(3 \times 10 \mathrm{ml})$. The organic layers were concentrated under reduced pressure to give crude 4,5-dihydro-1-cyclopentyl-4-(hydroxymethyl)imidazolin-2-one 13 as a pale brown oil which was used immediately without further purification; $v_{\max }\left(\mathrm{CHCl}_{3}\right)$ 3357, 1680; $\delta_{\mathrm{H}}\left(250 \mathrm{MHz} ; \mathrm{CDCl}_{3}\right)$ 1.3-1.9 $\left(8 \mathrm{H}, \mathrm{m}\right.$, cyclopentyl $\left.\mathrm{CH}_{2}\right), 3.15(1 \mathrm{H}, \mathrm{dd}, \mathrm{J} 9.3,5.9$ $\mathrm{Hz}), 3.45$ (2 H, m), $3.6(1 \mathrm{H}, \mathrm{m}), 3.75(1 \mathrm{H}, \mathrm{m}), 4.2(1 \mathrm{H}, \mathrm{q}, \mathrm{NCH}, \mathrm{J} 8.3 \mathrm{~Hz}), 4.4(1 \mathrm{H}, \mathrm{br}$, $\mathrm{OH}), 5.5$ (1 H, br s, NH).

The crude hydroxymethyl derivative 13 [from the reaction of the iodomethyl precursor 12 (294 mg, $1 \mathrm{mmol})]$ was dissolved in a solvent mixture of acetonitrile : water $(4: 1,10 \mathrm{ml})$ at $0{ }^{\circ} \mathrm{C}$. To this solution was added CAN (3.29 g, 6 equiv.) and the mixture was stirred at $0^{\circ} \mathrm{C}$ for $2 \mathrm{~h}$ before being allowed to reflux for $14 \mathrm{~h}$. The resulting solution was a pale green/yellow colour and after being extracted with $\mathrm{CHCl}_{3}(3 \times 20 \mathrm{ml})$ and dried $\left(\mathrm{MgSO}_{4}\right)$, the solvent was removed under reduced pressure. This gave an oily residue which solidified upon trituration with ether. This solid was purified using dry flash column chromatography (eluant light petroleum to ether) and finally recrystallised from a cyclohexane/ether mixture to give the title compound $\left(60.5 \mathrm{mg}, 36 \%\right.$ ) as a colourless solid, mp 149-150 ${ }^{\circ} \mathrm{C}$; (Found: $\mathrm{C}, 57.3$; H, 7.3; $\mathrm{N}, 16.6 ; \mathrm{C}_{8} \mathrm{H}_{12} \mathrm{~N}_{2} \mathrm{O}_{2}$ requires $\left.\mathrm{C}, 57.1 ; \mathrm{H}, 7.2 ; \mathrm{N}, 16.7 \%\right) ; v_{\max }\left(\mathrm{CHCl}_{3}\right) 3197,1767,1719,1456$, 1423, $1109 \mathrm{~cm}^{-1}$; ${ }_{\delta \mathrm{H}}(270 \mathrm{MHz}$; CDCl3) 1.4-2.0 (8 H, m, cyclopentyl CH2), 3.87 (2 H, s, 5-H), 4.2 (1 H, q, NCH, J 8 Hz), 9.18 (1 H, br s, NH); m/z 168 (M+, 60\%), 139 (100), 101 (94), 68 (59), 55 (20), 41 (51).

1-Cyclopentyl-4-methylimidazol-2-one 15. The iodomethylimidazolinone 12 (1.0 g, 3.4 mmol) was taken up in THF $(70 \mathrm{ml})$ and DBU $(1.47 \mathrm{ml}, 3$ equiv.) was added. This mixture was heated under reflux for $4 \mathrm{~h}$ before being left to cool. The precipitate was filtered off and the filtrate was evaporated under reduced pressure. The oily product was taken up into EtOAc 
$(20 \mathrm{ml})$ and washed once with brine $(20 \mathrm{ml})$ and after separating the layers the brine was back-extracted with EtOAc $(2 \times 20 \mathrm{ml})$. The combined organic layers were dried $\left(\mathrm{MgSO}_{4}\right)$ and the solvent was removed to give a buff solid. The solid was purified using dry flash column chromatography (eluant light petroleum:ether (1:1) to EtOAc) and gave the title compound (512 mg, 91\%) as a colourless solid, $\mathrm{mp} 154-156{ }^{\circ} \mathrm{C}$ (from cyclohexane); (Found: $\mathrm{C}, 64.8 ; \mathrm{H}, 8.6 ; \mathrm{N}, 16.6 . \mathrm{C}_{9} \mathrm{H}_{14} \mathrm{~N}_{2} \mathrm{O}$ requires $\left.\mathrm{C}, 65.0 ; \mathrm{H}, 8.5 ; \mathrm{N}, 16.9 \%\right) ; v_{\max }\left(\mathrm{CHCl}_{3}\right) 3172$, $1709,1670,1416 \mathrm{~cm}^{-1} ; \delta_{\mathrm{H}}\left(270 \mathrm{MHz} ; \mathrm{CDCl}_{3}\right) 1.4-1.9\left(8 \mathrm{H}, \mathrm{m}\right.$, cyclopentyl $\left.\mathrm{CH}_{2}\right), 2.0(3 \mathrm{H}, \mathrm{s}$, 4-Me), $4.4(1 \mathrm{H}, \mathrm{q}, \mathrm{NCH}, \mathrm{J} 7.3 \mathrm{~Hz}), 5.8(1 \mathrm{H}, \mathrm{s}, 5-\mathrm{H}), 10.5(1 \mathrm{H}, \mathrm{br} \mathrm{s}, \mathrm{NH}) ; \mathrm{m} / \mathrm{z} 166\left(M^{+}, 6 \%\right)$, 115 (26), 98 (100).

\section{Acknowledgements}

We thank GlaxoWellcome Research \& Development for their generous support of this research (studentship to P.A.H.).

\section{References}

1. For reviews see: (a) Cardillo, G.; Orena, M. Tetrahedron 1990, 46, 3321; (b) Harding, K. E.; Tiner, T. H. in Comprehensive Organic Synthesis; Trost, B. M.; Fleming, I. Eds.; Pergamon Press: Oxford, 1991; vol. 4, p 363.

2. Preliminary communication, Hunt, P. A.; May, C.; Moody, C. J. Tetrahedron Lett. 1988, 29, 3001.

3. Betancor, C.; León, E. I.; Prange, T.; Salazar, J. A.; Suárez, E. J. Chem. Soc., Chem. Commun. 1989, 450.

4. Freire, R.; León, E. I.; Salazar, J. A.; Suárez, E. J. Chem. Soc., Chem. Commun. 1989, 452.

5. Balko, T. W.; Brinkmeyer, R. S.; Terando, N. H. Tetrahedron Lett. 1989, 30, 2045.

6. Creeke, P. I.; Mellor, J. M. Tetrahedron Lett. 1989, 30, 4435.

7. Cardillo, G.; Orena, M.; Penna, M.; Sandri, S.; Tomasini, C. Tetrahedron 1991, 47, 2263.

8. Fujita, M.; Kitagawa, O., Suzuki, T.; Taguchi, T. J. Org. Chem. 1997, 62, 7330.

9. Borch, R. F.; Bernstein, M. D.; Durst, H. D. J. Am. Chem. Soc. 1971, 93, 2897.

10. Neville, R. G.; McGee, J. J. Can. J. Chem. 1963, 41, 2123.

11. Knapp, S.; Rodiques, K. E.; Levorse, A. T.; Ornaf, R. M. Tetrahedron Lett. 1985, 26, 1803.

12. Knapp, S.; Levorse, A. T. J. Org. Chem. 1988, 53, 4006.

13. Barluenga, J.; Canteli, R.-M.; Flórez, J. J. Org. Chem. 1994, 59, 602. 
14. Cook, G. R.; Barta, N. S.; Stille, J. R. J. Org. Chem. 1992, 57, 461. 